\title{
CHARACTERIZATION OF A CLASS OF GRAPHS WITH UNIQUE MINIMUM GRAPHOIDAL COVER
}

\author{
S. ARUMUGAM, INDRA RAJASINGH AND P. ROUSHINI LEELY PUSHPAM
}

\begin{abstract}
A graphoidal cover of a graph $G$ is a collection $\psi$ of (not necessarily open) paths in $G$ such that every vertex of $G$ is an internal vertex of at most one path in $\psi$ and every edge of $G$ is in exactly one path in $\psi$. The minimum cardinality of a graphoidal cover of $G$ is called the graphoidal covering number of $G$ and is denoted by $\eta$. Two graphoidal covers $\psi_{1}$ and $\psi_{2}$ of a graph $G$ are said to be isomorphic if there exists an automorphism $f$ of $G$ such that $\psi_{2}=\left\{f(P) / P \in \psi_{1}\right\}$. A graph $G$ is said to have a unique minimum graphoidal cover if any two minimum graphoidal covers of $G$ are isomorphic. In this paper we characterize the class of all graphs $G$ with a unique minimum graphoidal cover when $\delta=2$ and no end block of $G$ is a cycle.
\end{abstract}

\section{Introduction}

By a graph $G=(V, E)$ we mean a finite undirected, connected graph without loops. The order and size of $G$ are denoted by $p$ and $q$ respectively. For graph theoretic terminology we refer to Harary [6].

If $P=\left(v_{0}, v_{1}, \ldots, v_{n}\right)$ is a path or a cycle in $G, v_{1}, v_{2}, \ldots, v_{n-1}$, are called internal vertices of $P$. If $P=\left(v_{0}, v_{1}, \ldots, v_{n}\right)$ and $Q=\left(v_{n}=w_{0}, w_{1}, \ldots, w_{m}\right)$ are two paths in $G$ then the walk obtained by concatenating $P$ and $Q$ at $v_{n}$ is denoted by $P \circ Q$ and the path $\left(v_{n}, v_{n-1}, \ldots, v_{1}, v_{0}\right)$ is denoted by $P^{-1}$. For any subset $V_{1}$ of $V$ the subgraph of $G$ induced by $V_{1}$ is denoted by $\left\langle V_{1}\right\rangle$.

The concept of graphoidal cover was introduced by Acharya and Sampathkumar [1].

Definition 1.1. A graphoidal cover of a graph $G$ is a collection $\psi$ of (not necessarily open) paths in $G$ satisfying the following conditions.

(i) Every path in $\psi$ has at least two vertices.

(ii) Every vertex of $G$ is an internal vertex of at most one path in $\psi$.

(iii) Every edge of $G$ is in exactly one path in $\psi$.

The minimum cardinality of a graphoidal cover of $G$ is called the graphoidal covering number of $G$ and is denoted by $\eta$.

Received November 14, 2001; revised September 12, 2002.

2000 Mathematics Subject Classification. 05C.

Key words and phrases. Graphoidal cover, graphoidal covering number.

Research supported by DST Project DST/MS/064/96. 
Arumugam and Pakkiam introduced the concept of isomorphism between graphoidal covers of a graph. [5]

Definition 1.2. Two graphoidal covers $\psi_{1}$ and $\psi_{2}$ of a graph $G$ are said to be isomorphic if there exists an automorphism $f$ of $G$ such that $\psi_{2}=\left\{f(P) / P \in \psi_{1}\right\}$. A graph $G$ is said to have a unique minimum graphoidal cover if any two minimum graphoidal covers of $G$ are isomorphic.

An elaborate review of results in graphoidal covers with several interesting applications and a large collection of unsolved problems is given in [2].

Definition 1.3. Let $\psi$ be a collection of internally disjoint paths in $G$. A vertex of $G$ is said to be an interior vertex of $\psi$ if it is an internal vertex of some path in $\psi$. Any vertex which is not an interior vertex of $\psi$ is said to be an exterior vertex of $\psi$.

Theorem 1.4.([8]) For any graphoidal cover $\psi$ of $G$, let $t_{\psi}$ denote the number of exterior vertices of $\psi$. Let $t=\min t_{\psi}$, where the minimum is taken over all graphoidal covers of $G$. Then $\eta=q-p+t$.

Corollary 1.5.([8]) For any graph $G, \eta \geq q-p$. Moreover the following are equivalent.

(i) $\eta=q-p$

(ii) There exists a graphoidal cover without exterior vertices

(iii) There exists a set of internally disjoint and edge disjoint paths without exterior vertices.

Corollary 1.6.([8]) Let $G$ be a graph with $\eta=q-p$. Then the minimum vertex degree $\delta$ in $G$ is at least two.

Corollary 1.7.([8]) Let $G$ be a graph with $\eta=q-p$. Then the maximum vertex degree $\triangle$ in $G$ is at least three.

Theorem 1.8.([7]) For any graph $G$ with $\delta \geq 3, \eta=q-p$.

Let $\mathcal{F}$ denote the class of all connected graphs with $\eta=q-p$.

Theorem 1.9.([3]) Let $G$ be a 2-edge connected graph. Then $G \notin \mathcal{F}$ if and only if every block of $G$ is a cycle or a cycle with exactly one chord or a theta graph and at most one block of $G$ is not a cycle.

Theorem 1.10.([4]) Let $G$ be a connected graph with $\delta=2$ and $\kappa^{\prime}=1$. Then $G \notin \mathcal{F}$ if and only if there exists a cut edge e of $G$ such that at least one component of $G-e$ is a graph, all of whose blocks are cycles.

In [5], it has been proved that if a graph $G$ has a unique minimum graphoidal cover, then $\delta \leq 3$ and when $\delta=3, G$ has a unique minimum graphoidal cover if and only if $G=K_{4}$. Trees and unicyclic graphs having a unique minimum graphoidal cover have 
also been characterized [5]. In this paper we characterize the class of graphs with a unique minimum graphoidal cover when $\delta=2$, and no end block of $G$ is a cycle.

\section{Main Results}

Theorem 2.1. Let $G$ be a graph in which $\delta=2$ and no end block of $G$ is a cycle. Let $G^{\prime}$ be the graph (allowing multiple edges) obtained from $G$ by contracting all vertices of degree 2. If $G$ has a unique minimum graphoidal cover then $G^{\prime}$ also has a unique minimum graphoidal cover.

Proof. Suppose $G$ has a unique minimum graphoidal cover. If $\eta>q-p$, since no end block of $G$ is a cycle, it follows from Theorem 1.9 and Theorem 1.10 that $G$ is either a cycle with exactly one chord or a $\theta$-graph. In either of the cases there exist two nonisomorphic minimum graphoidal covers of $G$, which is a contradiction. Hence $\eta=q-p$. Now let $\psi$ be a minimum graphoidal cover of $G$ so that each vertex of $G$ is interior to $\psi$. As we contract the vertices of degree 2 in $G$ we perform the same sequence of opertaions on the paths in $\psi$. Let $\psi^{\prime}$ be the resulting collection. Then clearly $\psi^{\prime}$ is a minimum graphoidal cover of $G^{\prime}$. Now let $\psi_{1}$ and $\psi_{2}$ be two minimum graphoidal covers of $G^{\prime}$. Then there exist two minimum graphoidal covers $\psi_{3}$ and $\psi_{4}$ of $G$ such that $\psi_{3}^{\prime}=\psi_{1}$ and $\psi_{4}^{\prime}=\psi_{2}$. Since $G$ has a unique minimum graphoidal cover, there exists an automorphism $\alpha$ of $G$ such that $\psi_{4}=\left\{\alpha(P) / P \in \psi_{3}\right\}$. Let $\alpha_{\mid V\left(G^{\prime}\right)}=\alpha^{\prime}$. Clearly, $\alpha^{\prime}$ is an automorphism of $G^{\prime}$ and $\psi_{2}=\left\{\alpha^{\prime}(P) / P \in \psi_{1}\right\}$, so that $G^{\prime}$ has a unique minimum graphoidal cover.

We observe that $G^{\prime}$ is a multigraph with $\delta \geq 3$. We first characterize multigraphs with $\delta \geq 3$ having a unique minimum graphoidal cover.

Theorem 2.2. Let $G$ be a multigraph with $\delta \geq 3$. Then $G$ has a unique minimum graphoidal cover if and only if $G$ is isomorphic to one of the following graphs, $G_{i}, 1 \leq$ $i \leq 12$ given in Figure 1 .

Proof. It can be easily verified that each of the graphs $G_{i}, 1 \leq i \leq 12$, has a unique minimum graphoidal cover. Conversely suppose $G$ is a multigraph with $\delta \geq 3$ having a unique minimum graphoidal cover. Let $P_{1}=\left(u_{1}, u_{2}, \ldots, u_{n}\right)$ be a longest path in $G$ so that vertices adjacent to $u_{1}$ or $u_{n}$ are already in $P_{1}$. In the sequel all the graphoidal covers that are exhibited are minimal by Corollay 1.5 .

Case (i). $u_{1}$ and $u_{n}$ are non-adjacent.

Since $\delta \geq 3$ we can find vertices $u_{i}, u_{j}$ in $P_{1}$ such that $2 \leq i \leq j \leq n-1$, and $u_{i}, u_{j}$ are adjacent to $u_{1}$. Similarly we can find vertices $u_{r}, u_{s}$ in $P_{1}$ such that $2 \leq r \leq s \leq n-1$ and $u_{r}, u_{s}$ are adjacent to $u_{n}$. Now let $P_{2}=\left(u_{i}, u_{1} . u_{j}\right)$ and $P_{3}=\left(u_{r}, u_{n}, u_{s}\right)$. We claim that $j \leq r$. Suppose $j>r$.

If $i \neq j$, let

$$
Q_{1}=\left(u_{1}, u_{2}, \ldots, u_{j}\right),
$$




$$
\text { and } \quad \begin{aligned}
Q_{2} & =\left(u_{i}, u_{1}, u_{j}, u_{j+1}, \ldots, u_{n}, u_{r}\right), \\
\text { If } \quad Q_{3} & =\left(u_{s}, u_{n}\right) . \\
i & =j, \text { let } \\
Q_{1} & =\left(u_{i}, u_{1}, \ldots, u_{j}\right), \\
Q_{2} & =\left(u_{1}, u_{j}, u_{j+1}, \ldots, u_{n}\right), \text { and } \\
Q_{3} & =P_{3}
\end{aligned}
$$

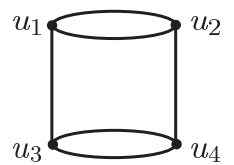

$G_{1}$

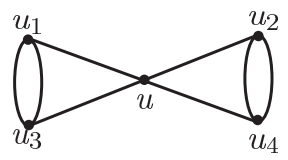

$G_{5}$

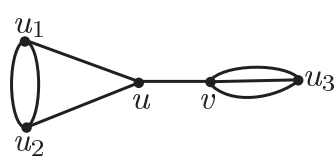

$G_{9}$

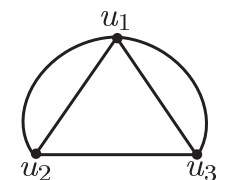

$G_{2}$

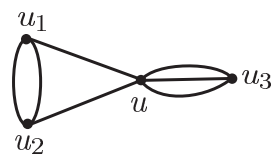

$G_{6}$

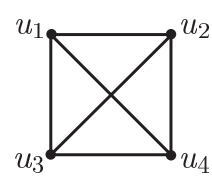

$G_{3}$

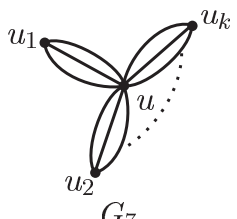

$G_{7}$

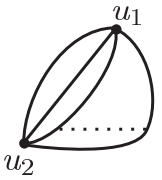

$G_{4}$

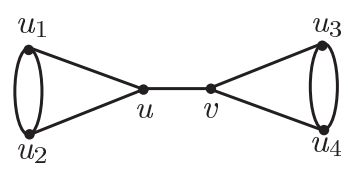

$G_{8}$

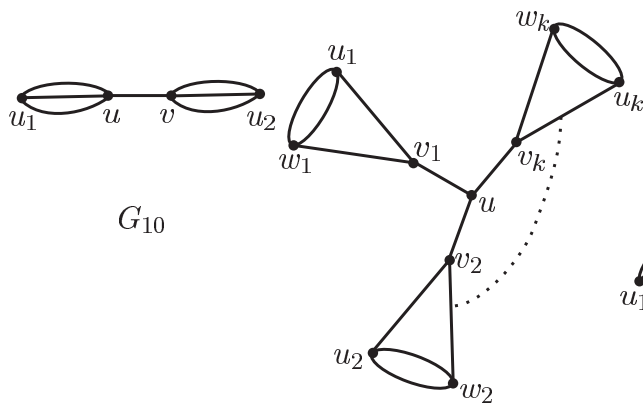

$G_{11}$

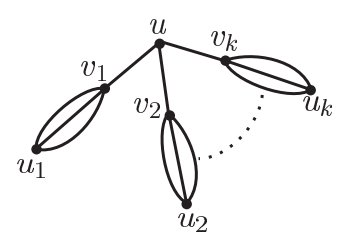

$G_{12}$

Figure 1.

Let $\mathcal{P}$ be a collection of paths in $G$ such that $\psi_{1}=\left\{P_{1}, P_{2}, P_{3}\right\} \cup \mathcal{P}$ and $\psi_{2}=$ $\left\{Q_{1}, Q_{2}, Q_{3}\right\} \cup \mathcal{P}$ are minimum graphoidal covers of $G$. Clearly $\psi_{1}$ and $\psi_{2}$ give rise to different partitions of $E(G)$ so that $\psi_{1}$ and $\psi_{2}$ are non-isomorphic, which is a contradiction. Hence $j \leq r$.

Now let $V(G)=\left\{u_{1}, u_{2}, \ldots, u_{n}\right\}, H=P_{1} \cup P_{2} \cup P_{3}$ and $H^{\prime}=\left\langle V\left(P_{1}\right)\right\rangle$. We prove that $H^{\prime}=H$. If not, there exists an edge $e=u_{l} u_{m} \in E\left(H^{\prime}\right) \backslash E(H)$ where $1 \leq l<m \leq n$. Since $u_{1}$ and $u_{n}$ are non-adjacent $\{l, m\} \neq\{1, n\}$. Suppose $m<n$.

If $m \neq 2$, Let

$$
Q_{1}=\left(u_{1}, u_{2}, \ldots, u_{m}\right)
$$


and

$$
\text { and } \quad \begin{aligned}
Q_{2} & =\left(u_{l}, u_{m}, u_{m+1}, \ldots, u_{n}\right) . \\
& \quad m=2, \text { let } \\
Q_{1} & =\left(u_{1}, u_{2}, u_{1}\right) \\
Q_{2} & =\left(u_{2}, u_{3}, \ldots, u_{m}\right)
\end{aligned}
$$

Then $\psi_{1}=\left\{P_{1}, P_{2}, P_{3}, e\right\}$ and $\psi_{2}=\left\{P_{2}, P_{3}, Q_{1}, Q_{2}\right\}$ are two nonisomorphic minimum graphoidal covers of $G$, which is a contradiction. Similarly if $l>1$ we get a contradiction. Hence $H^{\prime}=H$. Clearly $3 \leq p \leq 6$. When $p=3, G$ is isomorphic to $G_{7}$ with exactly two blocks. When $p=4, G$ is isomorphic to $G_{6}$ or $G_{10}$; when $p=5, G$ is isomorphic to $G_{5}$ or $G_{9}$ and when $p=6, G$ is isomorphic to $G_{8}$.

If $V(G) \neq\left\{u_{1}, u_{2}, \ldots, u_{n}\right\}$, let $w$ be a vertex not in $P_{1}$. Let $P_{4}=\left(w_{1}, w_{2}, \ldots, w, \ldots, w_{d}\right)$ be a longest path in $G$ containing $w$ and internally disjoint with the paths $P_{1}, P_{2}, P_{3}$ and with one of its ends say $w_{d}$, in $P_{1}$. Then $w_{d}=u_{k}$ for some $k, 1<k<n$. Let $R_{1}$ and $R_{2}$ be the $\left(u_{1}, u_{k}\right)$ and $\left(u_{k}, u_{n}\right)$-sections of $P_{1}$ [Refer Figure 2]. Let $l_{1}, l_{2}$ and $l_{3}$ be the lengths of the paths $R_{1}, R_{2}$ and $P_{4}$ respectively.

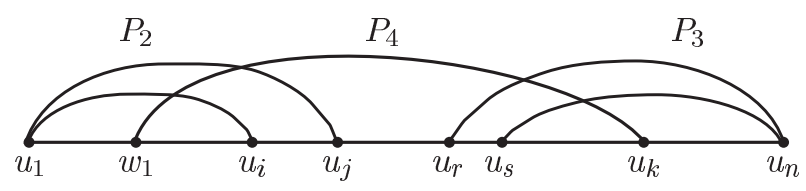

Figure 2.

If $w_{1} \in V\left(P_{1}\right)$, then there exists a collection of paths $\mathcal{P}$ in $G$ such that $\psi_{1}=$ $\left\{P_{1}, P_{2}, P_{3}, P_{4}\right\} \cup \mathcal{P}$ and $\psi_{2}=\left\{P_{2}, P_{3}, R_{1}, P_{4} \circ R_{2}\right\} \cup \mathcal{P}$ are minimum graphoidal covers of $G$ and $\psi_{1}$ and $\psi_{2}$ are nonisomorphic, which is a contradiction. Hence $w_{1} \notin V\left(P_{1}\right)$. Since $\delta \geq 3$, we can find vertices $w_{a}, w_{b}$ in $P_{4}$ such that $1 \leq a \leq b \leq d$ and $w_{a}$, $w_{b}$ are adjacent to $w_{1}$. Let $P_{5}=\left(w_{a}, w_{1}, w_{b}\right)$. Now we prove that $l_{1}=l_{2}=l_{3}$. There exists a collection $\mathcal{P}$ of paths in $G$ such that

and

$$
\begin{aligned}
\psi & =\left\{P_{1}, P_{2}, P_{3}, P_{4}, P_{5}\right\} \cup \mathcal{P} \\
\psi_{1} & =\left\{P_{2}, P_{3}, P_{5}, R_{1} \circ P_{4}^{-1}, R_{2}\right\} \cup \mathcal{P} \\
\psi_{2} & =\left\{P_{2}, P_{3}, P_{5}, P_{4} \circ R_{2}, R_{1}\right\} \cup \mathcal{P}
\end{aligned}
$$

are minimum graphoidal covers of $G$. The paths $P_{1}, P_{4}$ of $\psi$ are of lengths $l_{1}+l_{2}, l_{3}$ respectively, the paths $R_{1} \circ P_{4}^{-1}, R_{2}$ of $\psi_{1}$ are of lengths $l_{1}+l_{3}, l_{2}$ respectively and the paths $P_{4} \circ R_{2}, R_{1}$ of $\psi_{2}$ are of lengths $l_{3}+l_{2}, l_{1}$ respectively. Since $G$ has a unique minimum graphoidal cover we see that $l_{1}=l_{2}=l_{3}$. As before we can prove that $\left\langle V\left(P_{1} \cup P_{4}\right)\right\rangle=P_{1} \cup P_{2} \cup P_{3} \cup P_{4} \cup P_{5}$. If $V(G)=V\left(P_{1} \cup P_{4}\right)$ then $2 \leq n \leq 7$. Since $l_{1}=l_{2}, n$ is an odd number. If $n=7$ then $G$ is isomorphic to $G_{11}$. Let $n=5$. If $i=j$, $r=s, a=b$ then $G$ is isomorphic to $G_{12}$. Otherwise either $i \neq j$ or $r \neq s$ or $a \neq b$. Let $a \neq b$, then $w_{b}=w_{d}$ (Refer Figure 3). 
Let $Q_{1}=\left(w_{d}, w_{d-1}, \ldots, w_{1}, w_{d}\right)$ and $Q_{2}=\left(w_{1}, w_{2}\right)$. Then $\psi_{1}=\left(P_{1}, P_{2}, P_{3}, Q_{1}, Q_{2}\right\} \cup$ $\mathcal{P}$ and $\psi_{2}=\left\{P_{1}, P_{2}, P_{3}, P_{4}, P_{5}\right\} \cup \mathcal{P}$ are nonisomorphic minimum graphoidal covers of $G$, which is a contradiction. In a similar way we get contradictions when $r \neq s$ and $i \neq j$.

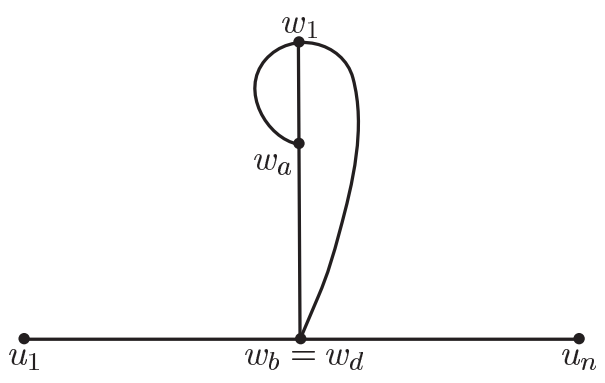

Figure 3.

Now if $n=3$ then $G$ is isomorphic to $G_{7}$. If $V(G) \neq V\left(P_{1} \cup P_{4}\right)$, then repeating the above process we see that $G$ is isomorphic to $G_{7}, G_{11}$ or $G_{12}$.

Case (ii) $u_{1}$ and $u_{n}$ are adjacent

Let $t$ be the number of edges joining $u_{1}$ and $u_{n}$. We prove that $V(G)=\left\{u_{1}, u_{2}, \ldots, u_{n}\right\}$. Suppose not, then there exists a vertex $w \notin V\left(P_{1}\right)$ such that $w$ is adjacent to some $u_{i}$ and hence $G$ contains a path of length greater than $n-1$, which is a contradiction. Hence $V(G)=\left\{u_{1}, u_{2}, \ldots, u_{n}\right\}$. Since $\delta \geq 3$ we can find vertices $u_{i}, u_{j}$ in $P_{1}$ such that $2 \leq i \leq n-1$ and $2 \leq j \leq n-1, u_{i}$ is adjacent to $u_{1}$ and $u_{j}$ is adjacent to $u_{n}$. Let $P_{2}=\left(u_{i}, u_{1}, u_{n}, u_{j}\right), H=P_{1} \cup P_{2}$ and $t=1$ (Refer Figure 4 ).

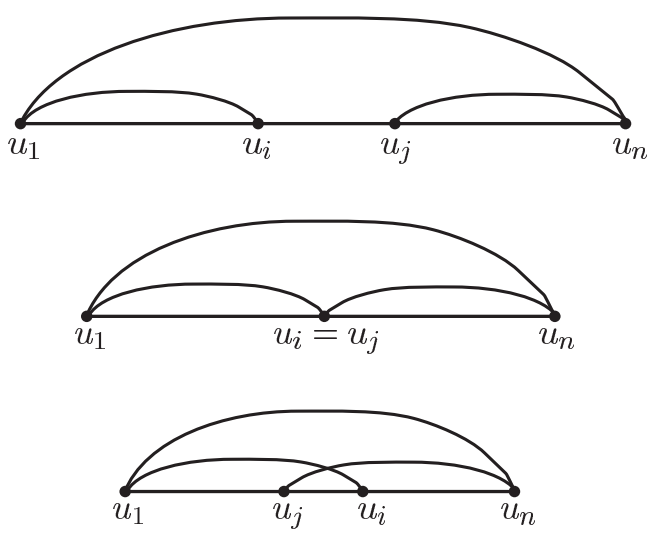

Figure 4.

We claim that $G=H$. If not, there exists an edge $e=u_{l} u_{m} \in E(G) \backslash E(H)$ where 
$1 \leq l<m \leq n$. Since $t=1,\{l, m\} \neq\{1, n\}$. Supoose $m<n$.

If $m \neq 2$, let

$$
\begin{aligned}
Q_{1} & =\left(u_{1}, u_{2}, \ldots, u_{m}\right) \\
\text { and } & Q_{2}=\left(u_{l}, u_{m}, u_{m+1}, \ldots, u_{n}\right) .
\end{aligned}
$$

If $m=2$, let

and

$$
\begin{aligned}
& Q_{1}=\left(u_{1}, u_{2}, u_{1}\right) \\
& Q_{2}=\left(u_{2}, u_{3}, \ldots, u_{n}\right) .
\end{aligned}
$$

Let $S$ denote the set of edges not covered by the paths $P_{1}$ and $P_{2}$. Then $\psi_{1}=$ $\left\{P_{1}, P_{2}\right\} \cup S$ and $\psi_{2}=\left\{P_{2}, Q_{1}, Q_{2}\right\} \cup(S \backslash\{e\})$ are nonisomorphic minimum graphoidal covers of $G$, which is a contradiction. Similarly if $l>1$ we get a contradiction. Hence $G=H$. Therefore when $i<j, G$ is isomorphic to $G_{1}$, when $i=j, G$ is isomorphic to $G_{2}$ and when $i<j, G$ is isomorphic to $G_{3}$.

Suppose $t=2, P_{2}=\left(u_{n}, u_{1}, u_{n}\right)$ and $H=P_{1} \cup P_{2}$. When $G=H, G$ is isomorphic to $G_{4}$ with $\delta\left(G_{4}\right)=3$. When $G \neq H$, there exists an edge $e \in E(G) \backslash E(H)$. Let $H_{1}=H \cup\{e\}$. Proceeding as before we can prove that $G=H_{1}$ and $G$ is isomorphic to $G_{1}$ or $G_{2}$. Let $t>2$. We claim that the length of $P_{1}$ is one. Suppose not, then there exists an edge $e_{1}=u_{l} u_{m} \in E(G) \backslash E(H)$ where $1 \leq l \leq m \leq n,\{l, m\} \neq\{1, n\}$. Since $t>2$, there exists an edge $e_{2}=u_{1} u_{n}$ which does not lie on $P_{2}$ (Refer Figure 5 ). Let $S$ be the set of edges not covered by the paths $P_{1}, P_{2}$, and the edges $e_{1}$ and $e_{2}$. Let $\psi_{1}=\left\{\left(u_{1} u_{2} \cdots u_{n} u_{1}\right), P_{2}, e_{1}\right\} \cup S$

$$
\text { and } \quad \psi_{2}= \begin{cases}\left\{\left(P_{1},\left(u_{1} u_{n} u_{1}\right),\left(u_{m} u_{1} u_{n}\right)\right\} \cup S\right. & \text { if } 1=l<m<n \\ \left\{\left(P_{2},\left(u_{1} u_{m} u_{m+1} \cdots u_{n} u_{1}\right),\left(u_{1} u_{2} \cdots u_{m}\right)\right\} \cup S\right. & \text { if } 1<l<m<n\end{cases}
$$

Then $\psi_{1}$ and $\psi_{2}$ are minimum graphoidal covers which are nonisomorphic, which is a contradiction. Hence $G$ is isomorphic to $G_{4}$ with $\delta\left(G_{4}\right)>3$.

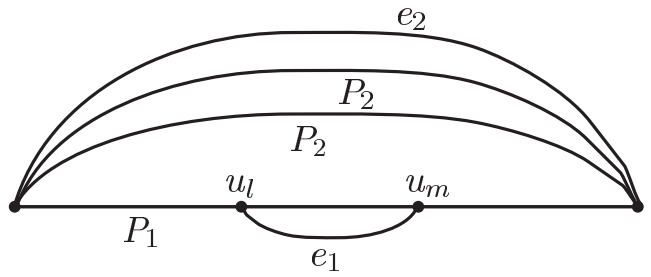

Figure 5.

Now we define a series of graphs corresponding to the graphs $G_{i}$ given in Figure 1 as follows:

For any edge $u v$, let $(u v)^{m}$ denote the $(u, v)$-path obtained by subdividing $m$ times the edge $u v$. 
Definition 2.3. Let $H_{1}$ be the graph obtained from $G_{1}$ by replacing the multiple edges $u_{1} u_{2}$ by $\left(u_{1} u_{2}\right)^{m}$, the multiple edges $u_{3} u_{4}$ by $\left(u_{3} u_{4}\right)^{n}$, the edge $u_{3} u_{1}$ by $\left(u_{3} u_{1}\right)^{a}$, and the edge $u_{2} u_{4}$ by $\left(u_{2} u_{4}\right)^{b}$ where $m, n \geq 1$ and $a, b \geq 0$. Let $H_{2}$ be the graph obtained from $G_{2}$ by replacing the multiple edges $u_{1} u_{2}$ by $\left(u_{1} u_{2}\right)^{m}$, the multiple edges $u_{1} u_{3}$ by $\left(u_{1} u_{3}\right)^{n}$ and the edge $u_{2} u_{3}$ by $\left(u_{2} u_{3}\right)^{a}$ where $m, n \geq 1$ and $a \geq 0$.

Let $H_{3}, H_{4}$ be the graphs obtained from $G_{3}$ and $G_{4}$, respectively by replacing each edge $u v$ by $(u v)^{m}, m \geq 1$.

Let $H_{5}$ be the graph obtained from $G_{5}$ by replacing the multiple edges $u_{1} u_{2}$ by $\left(u_{1} u_{2}\right)^{m}$, the multiple edges $u_{3} u_{4}$ by $\left(u_{3} u_{4}\right)^{n}$, the edges $u u_{1}, u u_{2}$ by $\left(u u_{1}\right)^{a},\left(u u_{2}\right)^{a}$ respectively and the edges $u u_{3}, u u_{4}$ by $\left(u u_{3}\right)^{b},\left(u u_{4}\right)^{b}$ respectively, where $m, n \geq 1$ and $a, b \geq 0$.

Let $H_{6}$ be the graph obtained from $G_{6}$ by replacing the multiple edges $u_{1} u_{2}$ by $\left(u_{1} u_{2}\right)^{m}$, the multiple edges $u u_{3}$ by $\left(u u_{3}\right)^{n}$ and the edges $u u_{1}, u u_{2}$ by $\left(u u_{1}\right)^{a},\left(u u_{2}\right)^{a}$ respectively, where $m, n \geq 1$ and $a \geq 0$.

When $\Delta\left(G_{7}\right)>6$, let $H_{7}$ be the graph obtained from $G_{7}$ by replacing the multiple edges $u u_{1}$ by $\left(u u_{1}\right)^{m}$ and $u u_{2}$ by $\left(u u_{2}\right)^{n}, m, n \geq 1$. When $\Delta\left(G_{7}\right)=6$ let $H_{7}$ be the graph obtained from $G_{7}$ by replacing each edge $u v$ by $(u v)^{m}, m \geq 1$.

Let $H_{8}$ be the graph obtained from $G_{8}$ by replacing the multiple edges $u_{1} u_{2}$ by $\left(u_{1} u_{2}\right)^{n}$, the multiple edges $u_{3} u_{4}$ by $\left(u_{3} u_{4}\right)^{m}$, the edges $u_{1} u, u_{2} u$ by $\left(u_{1} u\right)^{a},\left(u_{2} u\right)^{a}$ respectively and the edges $v u_{3}, v u_{4}$ by $\left(v u_{3}\right)^{b},\left(v u_{4}\right)^{b}$ respectively and the edges $u v$ by $(u v)^{c}$ where $m, n \geq 1$ and $a, b, c \geq 0$.

Let $H_{9}$ be the graph obtained from $G_{9}$ by replacing the multiple edges $u_{1} u_{2}$ by $\left(u_{1} u_{2}\right)^{n}$, the multiple edges $v u_{3}$ by $\left(v u_{3}\right)^{m}$, the edges $u_{1} u, u_{2} u$ by $\left(u_{1} u\right)^{a},\left(u_{2} u\right)^{a}$ respectively and the edges $u v$ by $(u v)^{b}$, where $m, n \geq 1$ and $a, b \geq 0$.

Let $H_{10}$ be the graph obtained from $G_{10}$ by replacing the multiple edges $u_{1} u$ by $\left(u_{1} u\right)^{m}$, the multiple edges $u_{2} u$ by $\left(u_{2} u\right)^{n}$, and the edge $u v$ by $(u v)^{a}$ where $m, n \geq 1$ and $a \geq 0$.

Let $H_{11}$ be the graph obtained from $G_{11}$ by replacing the multiple edges $u_{i} w_{i}, 1 \leq$ $i \leq k$, by $\left(u_{i} w_{i}\right)^{m}$, the edges $u v_{i}, 1 \leq i \leq k$, by $\left(u v_{i}\right)^{a}$, the edges $u_{i} v_{i}, w_{i} v_{i}, 1 \leq i \leq k$, by $\left(u_{i} v_{i}\right)^{b},\left(w_{i}, v_{i}\right)^{b}$ respectively, where $m \leq 1$ and $a, b \leq 0$.

Let $H_{12}$ be the graph obtained from $G_{12}$ by replacing the multiple edges $u_{i} v_{i}, 1 \leq$ $i \leq k$, by $\left(u_{i} v_{i}\right)^{m}$ the edges $u v_{i}, 1 \leq i \leq k$, by $\left(u v_{i}\right)^{a}$ where $m \geq 1$ and $a \geq 0$.

The following theorem gives a characterization of simple graphs $G$ with unique minimum graphoidal cover and with $\delta=2$ in which no end block of $G$ is a cycle.

Theorem 2.4. Let $G$ be a simple graph with $\delta=2$ in which no end block is a cycle. Then $G$ has a unique minimum graphoidal cover if and only if $G$ is not a $\theta$-graph and $G$ is isomorphic to one of the graphs $H_{i}, 1 \leq i \leq 12$ given in Definition 2.3 .

Proof. Suppose $G$ has a unique minimum graphoidal cover. Clearly $G$ is not a $\theta$-graph. Let $G^{\prime}$ be the graph obtained by contracting all vertices of degree 2 in $G$. By Theorem $2.1 G^{\prime}$ has a unique minimum graphoidal cover. By Theorem $2.2 G^{\prime}$ is isomorphic to one of the graphs $G_{i}, 1 \leq i \leq 12$ given in Figure 1. We claim that if $G^{\prime}$ is isomorphic to $G_{i}$, then $G$ is isomorphic to $H_{i}$. 
Let $G^{\prime}$ be isomorphic to $G_{1}$. Since any automorphism of $G_{1}$ maps $u_{1}, u_{3}$ to $u_{2}, u_{4}$ or $u_{1}, u_{2}$ to $u_{3}, u_{4}$ it follows that the multiple edges $u_{1} u_{2}$ must be subdivided the same number of times and the multiple edges $u_{3} u_{4}$ must be subdivided the same number of times to obtain $G$. Hence $G$ is isomorphic to $H_{1}$. The proof is similar if $G^{\prime}$ is isomorphic to $G_{i}, 2 \leq i \leq 12$.

Conversely suppose $G$ is not a $\theta$-graph and $G$ is isomorphic to $H_{i}, 1 \leq i \leq 12$. Let $\psi_{i}$ be a minimum graphoidal cover of $G_{i}$. $H_{i}$ is obtained from $G_{i}$ by subdivding the edges of $G_{i}$ as given in Definition 2.3. Perform the same sequence of subdivisions on the edges of the paths in $\psi_{i}$. Let $\psi_{i}^{\prime}$ be the resulting collection of paths. Then $\psi_{i}^{\prime}$ is a minimum graphoidal cover of $H_{i}$ and any minimum graphoidal cover of $H_{i}$ is isomorphic to $\psi_{i}^{\prime}$, $1 \leq i \leq 12$. Hence $H_{i}, 1 \leq i \leq 12$, has a unique minimum graphoidal cover.

\section{Acknowledgement}

We thank the referee for his excellent comments and valuable suggestions which led to substantial improvement of this paper.

\section{References}

[1] B. D. Acharya and E. Sampathkumar, Graphoidal covers graphoidal covering number of a graph, Indian J. Pure Appl. Math. 18(1987), 882-890.

[2] S. Arumugam, B. D. Acharya and E. Sampathkumar, Graphoidal covers of a graph a creative review, Graph Theory and its Applications, Proceedings of the National Workshop, Manonmaniam Sundaranar University, Tirunelveli, Eds. S. Arumugam, B.D. Acharya and E. Sampathkumar, Tata-McGraw Hill (1997), 1-28.

[3] S. Arumugam, Indra Rajasingh, R. Bharathi and P. Roushini Leely Pushpam, On graphs whose graphoidal covering number is one less than its cyclomatic number, Graph Theory and its Applications, Proceedings of the National Workshop, Manomaniam Sundaranar University, Tirunelveii, Eds. S. Arumugam, B. D. Acharya and E. Sampathkumar, TataMcGraw Hill (1997), 29-34.

[4] S. Arumugam, Indra Rajasingh and P. Roushini Leely Pushpam, A note on the graphoidal covering number of a graph, submitted for publication.

[5] S. Arumugam and C. Pakkiam, Graphs with unique minimum graphoidal cover, Indian J. Pure Appl. Math. 25(1994), 1147-1153.

[6] F. Harary, Graph Theory, Addison Wesley, Reading Mass, 1969.

[7] C. Pakkiam and S. Arumugam, On the graphoidal covering number of a graph, Indian J. Pure Appl. Math. 20(1989), 330-333.

[8] C. Pakkiam and S. Arumugam, The graphoidal covering number of unicyclic graphs, Indian J. Pure Appl. Math. 23(1992), 141-143.

Department of Mathematics, Manonmaniam Sundaranar University, Tirunelveli 627 012, India. Department of Mathematics, Loyola College, Chennai - 600 034, India.

Department of Mathematics, D. B. Jain College, Chennai - 600 096, India. 